\title{
Le cercle de craie : la formation des enseignants en Grèce
}

The chalk circle: Teacher training in Greece

El círculo de tiza: la formación de los educadores en Grecia

Elena Théodoropoulou et Alexandros Théodoridis

\section{OpenEdition}

Journals

Édition électronique

URL : http://journals.openedition.org/ries/964

DOI : $10.4000 /$ ries.964

ISSN : 2261-4265

Éditeur

Centre international d'études pédagogiques

Édition imprimée

Date de publication : 1 décembre 2010

Pagination : 119-128

ISBN : 978-2-8542-583-1

ISSN : $1254-4590$

\section{Référence électronique}

Elena Théodoropoulou et Alexandros Théodoridis, « Le cercle de craie : la formation des enseignants en Grèce », Revue internationale d'éducation de Sèvres [En ligne], 55 | décembre 2010, mis en ligne le 01 décembre 2013, consulté le 14 novembre 2019. URL : http://journals.openedition.org/ries/964 ; DOI : 10.4000/ries.964

(c) Tous droits réservés 


\section{Le cercle de craie : la formation des enseignants en Grèce*}

\section{Elena Théodoropoulou Alexandros Théodoridis}

\section{POSTULATS}

La qualité de l'éducation dépend d'abord de la manière dont l'enseignant comprend et assume son rôle. Sa formation doit relever d'un processus continu permettant d'intégrer de manière rationnelle et dynamique ses expériences professionnelles ainsi que personnelles et sociales. Elle doit s'inscrire dans un projet global et cohérent associant la philosophie de l'éducation portée par l'État, les théories de l'éducation issues de la recherche internationale et la philosophie de l'éducation propre à chaque individu. Elle doit viser une professionnalisation basée sur des capacités de métacognition, de réflexivité et de conscientisation de la part de l'éducateur.

C'est en ce sens qu'on peut considérer la question de la formation des enseignants comme exemplaire et emblématique car elle permet d'approcher, dans chaque pays, la réalité de l'éducation et de ses enjeux. Toute analyse critique d'un système de formation des enseignants révèle le corps de présuppositions idéologiques, politiques, scientifiques, philosophiques et pédagogiques sur lequel il se fonde. Cette analyse critique permet également d'expliciter la philosophie de l'éducation sous-jacente et de préciser ses conséquences sur l'éducation et la société. La formation continue des enseignants ne reflète pas seulement un besoin de chaque être humain, par ailleurs reconnu dès Platon; elle exprime la conception qu'a toute société de son système d'éducation. L'éducation, en tant que projet anthropologique et existentiel, idéologique et politique implique totalement les éducateurs qui interviennent de manière décisive sur les évolutions des générations futures. Si la question de l'éducation est cruciale pour la vie et la survie des sociétés et de leurs membres, on devrait donc renverser le processus de construction du système de formation des enseignants. Au lieu de monter une ingénierie éducative qui répond à des besoins souvent périphériques ou étrangers au phénomène intrinsèque de l'éducation (en essayant, a posteriori, de formaliser les principes qui soutiennent cette ingénierie), il conviendrait de préciser en premier lieu ces principes d'une manière qui dépasse la rhétorique

* Remerciements à Anne-Marie Bardi pour sa relecture de cet article (Ndlr). 
de l'évident et l'éthique de la soumission préalable aux conditions externes. Surtout, on devrait en premier lieu clarifier et déterminer le sens même de l'éducation.

Aujourd'hui, en Grèce, la construction du mécanisme de formation des enseignants n'évite pas le piège d'une logique tracée par les conseils de Bologne et de Lisbonne (1999-2000), ainsi que par ceux de l'Union européenne qui ont suivi ${ }^{1}$. Selon cette logique, l'éducateur doit acquérir une compétence lui permettant une redéfinition constante et rapide de son rôle, chaque fois que l'urgence l'exige, dans des environnements modernes sociopolitiques et économiques dénués de stabilité ; il doit adapter ses outils et ses objectifs aux exigences de la société dite de la connaissance ${ }^{2}$. Cet éducateur européen doit savoir abandonner ou dépasser son rôle traditionnel, désormais inadapté à ce processus de redéfinition et de conscientisation permanents ${ }^{3}$.

En Grèce, le projet actuel de formation des enseignants du primaire et du secondaire se développe sans états d'âmes sur la base contraignante des principes et des valeurs définissant les sociétés modernes européennes. Or ceci pose question : est-ce l'exercice de la pensée critique et créative qui intéresse la formation ou plutôt l'acquisition de la compétence d'adaptation à travers ces modalités de pensée ? Même si cette adaptation est justifiée par l'invocation du principe d'universalité (qui est le principe d'une éducation progressiste et égalitaire), ce même principe est en fait réduit à un appareil de normalisation qui dénature et morcelle l'humain, au lieu de l'envisager dans sa liberté et toute sa pluralité.

La formation, chaque fois qu'elle est conçue comme un mécanisme standardisé de réponse à une série de besoins standardisés, doit redevenir une question hautement philosophique, qui poserait à nouveau la question de l'être humain - en ce qui le constitue - et de ses besoins.

\section{LE PROJET GREC DE FORMATION DES ENSEIGNANTS Le système éducatif}

Le système éducatif grec comporte trois niveaux d'études : le premier, le second et le troisième degrés.

\footnotetext{
1. Commission des Communautés européennes, Rapport de la Commission. Les objectifs concrets d'éducation, COM (2001) 59 final, Bruxelles : 2001, 28 p. ; Commission des Communautés européennes, Mettre en œuvre le Programme communautaire de Lisbonne. Proposition de recommandation du Parlement européen et du Conseil établissant le cadre européen des certifications pour l'apprentissage tout au long de la vie, COM (2006) 479 final, Bruxelles : 2006, 23 p.

2. Commission européenne, Principes communs concernant les compétences et les qualifications des enseignants, Bruxelles : Commission européenne, 2005, 6 p., http://ec.europa.eu/education/policies/2010/doc/principles_fr.pdf 3. Le texte d'orientation politique lancé par le ministère de l'Éducation, de l'Éducation tout au long de la vie et des Religions a été soumis au débat public sur internet (Vers une politique nouvelle pour l'éducation tout au long de la vie en Grèce) ainsi que le texte Je vieillis en apprenant toujours. Synopse des principes du projet de Loi pour le développent de l'éducation tout au long de la vie, voir : http://www.opengov.gr/ypepth/ (en grec).
} 
Le premier degré comprend l'éducation préscolaire et l'éducation primaire. Depuis 2007, la scolarité dans les écoles maternelles est obligatoire pour les enfants qui ont cinq ans révolus; l'éducation primaire, qui dure six années, scolarise les enfants de six à douze ans.

L'éducation de second degré se divise en deux cycles d'études: celui, obligatoire, correspondant aux trois années de gymnase à la fin desquelles s'achèvent les dix années d'enseignement obligatoire prévues par la loi et celui, optionnel, incluant deux types d'écoles : le lycée général et le lycée professionnel. Dans l'éducation secondaire, on trouve également les écoles professionnelles qui, avec les lycées professionnels, constituent l'éducation professionnelle secondaire. Les études dans les écoles publiques du primaire et du secondaire sont gratuites.

L'éducation postsecondaire (mais n'appartenant pas à l'éducation de troisième degré) est assurée par les instituts de formation professionnelle octroyant un certificat de formation professionnelle, qui donne la possibilité de travailler dans le public et dans le privé.

L'éducation de troisième degré se divise depuis 2001 entre "éducation universitaire » assurée par les universités, les écoles polytechniques et l'École supérieure des beaux-arts et «éducation technologique » assurée par les institutions éducatives technologiques et l'École supérieure d'éducation pédagogique et technologique. Depuis l'année 1997-1998 fonctionne également l'Université grecque ouverte (éducation à distance). Le cycle d'études avant le master dure de quatre à cinq ans. L'entrée des étudiants dans l'éducation de troisième degré se fait selon un système d'examens écrits panhélleniques passés après la sortie du lycée et les études sont en principe gratuites, à quelques exceptions près, comme pour certains programmes postuniversitaires (master) ou les études à l'Université grecque ouverte.

Les enseignants du préscolaire et du primaire effectuent une formation initiale de huit semestres au sein de départements universitaires pédagogiques spécifiques. Les enseignants du secondaire suivent leurs études au sein de départements universitaires spécialisés par discipline. Le succès au cycle d'études prévu donne accès à l'exercice du métier dans le public et dans le privé ; ceux qui souhaitent enseigner dans le public s'inscrivent au concours (examens écrits panhélleniques) organisé par le Conseil supérieur de choix du personnel (ASEP). La réussite au concours permet d'être nommé dans le secteur public.

Les programmes de formation continue prévus par le ministère sont ouverts à tous les enseignants de l'enseignement public; ils visent au renforcement et à l'actualisation de la compétence scientifique et professionnelle des enseignants ainsi qu'à la revalorisation qualitative de l'école. Pourtant, les activités de formation actuellement développées ne sont pas effectuées en liaison avec les départements pédagogiques universitaires responsables de la formation initiale des enseignants. C'est le ministère (ou des agents dépendant du ministère) qui s'en charge. 


\section{Bref historique de la formation continue}

Le cadre institutionnel définissant les activités de formation continue depuis les années soixante-dix ne tend nullement à faire système, bien que cela soit revendiqué par les textes ${ }^{4}$.

De 1977 à 1992, tout effort de systématisation de la formation continue est sous le contrôle du ministère. En 1985 sont créés les premiers centres de formation décentralisée, les «centres de formation périphériques " (PEK). La formation prend à cette époque trois formes: initiale pour les enseignants nouvellement nommés, annuelle pour les enseignants ayant déjà cinq années de service, périodique et accélérée durant l'année scolaire et centrée sur les nouveautés éducatives. À partir de 1992, le contrôle du ministère demeurant fort, le rôle de l'Institut pédagogique est revalorisé : il a la responsabilité de la création et de la réalisation de la politique de formation ainsi que de la mise en place des programmes de formation. Cependant, ces activités ne sont pas suffisamment liées à la formation initiale et à la recherche ${ }^{5}$; de plus, les thématiques auxquelles les éducateurs voudraient être formés ne sont pas en adéquation avec celles qui sont proposées. De 1995 à 1999, les efforts pour alléger le centralisme ministériel et consolider l'institution de formation s'intensifient; en parallèle, on essaie d'inventorier les besoins de formation exprimés par les enseignants ${ }^{6}$. Jusqu'à présent, de multiples programmes de formation, obligatoires ou facultatifs, ont vu le jour pour des groupes variés d'enseignants; la formation des enseignants du primaire est faite dans les «centres d'enseignement» (Didaskalia), qui sont des écoles organisées et dirigées par les départements universitaires pédagogiques ${ }^{7}$.

Malgré ces évolutions, les efforts pour institutionnaliser la formation des maîtres au cours de cette période souffrent de faiblesses : discontinuité entre les diverses activités, centralisation des actions, centration des projets sur l'école, chevauchement des fonctions des agents impliqués, multiplicité injustifiable des objets cognitifs, difficulté d'associer théorie et pratique, non application des principes relatifs à l'éducation des adultes, faiblesse du matériel éducatif, indifférence aux besoins réels des enseignants ${ }^{8}$. Ce qui est beaucoup plus significatif,

\footnotetext{
4. Institut pédagogique, Proposition pour la formation des éducateurs, Athènes (2009) : p. 6 [http://www.pischools.gr/paideia_dialogos/prot_epimorf.pdf].

5. Ibid. et Institut Pédagogique, Formation des éducateurs, pp. 362-363, [http://www.pi-schools.gr/download/ programs/erevnes/.../s_357_390.pdf].

6. Ibid. et [http://www. pi-schools.gr/paideia_dialogos/prot_epimorf.pdf] (p. 7-8).

7. En 2002 fut créé l'Organisme de formation des éducateurs (OEPEK), suite à une demande persistante du monde éducatif : l'OEPEK se trouve sous l'autorité du ministre et son rôle consistait à constituer des propositions, à matérialiser et coordonner la politique de formation, à organiser des colloques et séminaires à caractère formateur. Pourtant, cet organisme resta à peu près inactif, hormis la réalisation de deux programmes de formation rapides accompagnés de matériel éducatif (Liaison entre l'environnement familial-social-culturel et la performance scolaire de l'enfant et Approches didactiques modernes pour le développement de la pensée critique-créative : 2007, V. Koulaïdis (coord. scient.), Athènes. [www.edugate.gr/.../sygxrones-didaktikes-proseggiseis-gia-tin-anaptyksi-kritikis-dimioyrgikis-skepsis].

8. Institut pédagogique, 2009, p. 7-8.
} 
c'est l'absence de tout texte programmatique, d'une philosophie (dans le sens péjoratif du terme) concernant ces efforts. On comprend, entre les lignes, que la formation se tient aux marges de la politique éducative et qu'elle est simplement considérée comme un complément du savoir ou un ajustement aux nouvelles orientations prescrites par le ministère.

\section{LA RÉFORME}

Récemment, le ministère de l'Éducation, de l'Éducation tout au long de la vie et des Religions a lancé un projet de restructuration de l'éducation centré sur l'école. Sous le titre L'École Nouvelle: en premier lieu, l'élève ${ }^{9}$, ce projet prévoit également un Programme de formation majeure des enseignants. Le projet touche plusieurs domaines - les connaissances scientifiques, les méthodes pédagogiques, les nouvelles technologies et l'administration - et se présente comme un projet intégrant la formation pédagogique de l'ensemble des enseignants. Se développant sur la base de l'expérience acquise, des résultats de recherches scientifiques et de l'expérience internationale, il prend en compte les résultats d'un débat public sur Internet et l'identification des besoins de formation exprimés par les enseignants, ce qui lui confère un caractère participatif et démocratique.

Ce Programme de formation majeure des enseignants est basé sur les principes de la formation des adultes : activités optionnelles, participation active des enseignants, flexibilité dans l'espace, le temps et le rythme et constitution d'environnements d'interaction sociale. Il se développe selon quatre axes: la didactique et la pratique, la méthodologie de l'éducation d'adultes, la méthodologie de l'éducation à distance, la réflexion sur l'application des sujets de formation à l'environnement de la classe.

La formation des enseignants est considérée comme l'un des piliers de l'école nouvelle, assurant la cohérence et la faisabilité de l'ensemble, les autres domaines clés étant les infrastructures, les méthodes didactiques, les curricula et l'évaluation.

Parmi les objectifs du projet d'école nouvelle on peut citer $^{10}$ :

- le développement chez l'élève de capacités horizontales : créativité, pensée critique, communication, collaboration, organisation, familiarisation avec les arts, prise d'initiatives, autonomie, esprit collectif social et conscience environnementale; l'élève doit devenir progressivement un "petit intellectuel, scientifique, chercheur », un polyglotte, un citoyen de son pays et du monde qui saura également « apprendre à apprendre »;

- la transformation de l'institution scolaire en une communauté d'apprentissage collaborative ;

9. Voir : www.ypepth.gr/el_ec_category12785.htm/.

10. Ibid. p. 8-9. 
- la promotion de l'usage des technologies de l'information et de communication (TIC) pour l'enseignement et le développement de l'enseignement des langues étrangères ;

- l'amélioration des relations et de la communication entre enseignants et élèves ;

- la prise en compte par les enseignants des besoins psychiques, sociaux et intellectuels des enfants à tous leurs stades de développement et une attention aux différences et aux situations spécifiques;

- la promotion de didactiques multiples et diversifiées.

Toutefois, les caractéristiques des sociétés occidentales modernes influent sur les objectifs stratégiques pour l'éducation tels qu'ils sont retenus au niveau de l'Union européenne. La conception européenne de la réalité personnelle, sociale et professionnelle - adaptation rapide à un monde en mouvement, émergence de nouvelles valeurs, règles de conduite et de communication - prend vite un caractère contraignant pour l'éducation telle que ce projet la conçoit. C'est dans ce cadre donc, que l'" école nouvelle " aspire à former des citoyens démocratiques capables d'une pensée critique et créative. Enseignants et étudiants devront "être encouragés à agir par eux-mêmes et à gérer d'une manière critique et créative le volume énorme d'information disponible afin de devenir à leur tour producteurs de contenus reflétant leurs propres valeurs et leurs priorités ${ }^{11}$.

Pour la première fois, on dispose d'un texte programmatique qui expose les intentions et les directives pour la formation et qui tente de théoriser d'une manière plus ou moins claire et décisive les tendances officielles. Néanmoins, il est difficile de savoir s'il s'agit d'une orientation pour l'éducation des élèves ou d'une orientation pour la formation des enseignants, ce qui pose, entre autres, la question de la distinction conceptuelle et philosophique entre les différents niveaux d'éducation et de formation. Ce qui saute immédiatement aux yeux, c'est que ces bouts de théorie font partie d'un "sens commun » formé par une terminologie largement répandue et systématiquement ressassée au cours des dernières décennies, un sens commun qui n'assure pas que la logique interne de cette théorie soit vraiment comprise. Quoi qu'il en soit, cette répétition permanente paraît avoir déjà créé un terrain propice à l'acceptation des principes sur lesquels se base le programme de formation.

\section{UNE PHILOSOPHIE MOLLE}

La conception de la formation des enseignants en Grèce est cohérente avec l'évolution des conceptions et des choix politiques. Elle reflète les tendances et les priorités de la société grecque en matière d'objectifs pédagogiques. Par le passé, la demande de démocratisation de la société et celle de l'éducation étaient

11. Voir : http://www.opengov.gr/ypepth (Cadre de délibération, p. 4). 
des objectifs majeurs. Aujourd'hui, la demande est de légitimer la « société de la connaissance ${ }^{12}$. Présenter la pensée réflexive comme un objectif du projet n'est que pure apparence; l'élaboration du projet résulte d'une réitération multiple de consignes issues des textes européens et ne laisse guère place à la réflexion individuelle, même si la pensée réflexive devient la plateforme conceptuelle de toute la philosophie de la formation. Il est intéressant que les références à la pensée réflexive ainsi qu'à d'autres éléments formant la philosophie de l'école nouvelle semblent renvoyer à la théorie des "écoles intelligentes " ${ }^{13}$. Il ne reste aux enseignants et aux élèves qu'à apprendre à gérer de manière créative le volume énorme des savoirs et des informations disponibles, cette sorte de créativité représentant leur seule marge de liberté dans l'exercice de leur pensée réflexive. La recherche des causes qui ont fait que le monde présent apparaît comme le seul possible ne figure pas parmi les préoccupations de cette éducation. Alors même que la question du rapport entre l'être et l'apparence est l'un des principaux enjeux de la formation, être et apparence sont condensés dans l'apparition énigmatique d'une "société du savoir».

C'est vrai, que selon ce projet, l'éducateur européen doit acquérir une capacité métacognitive qui lui assignerait la qualité de chercheur. Néanmoins, le discours ambiant - qui se veut un diagnostic neutre, voire clinique - affirme qu'on ne peut que nager dans l'eau tiède de la société de la connaissance et reconnaît la difficulté de l'entreprise mais non la complexité elle-même (ni les théories qui en découlent); il passe sous silence le risque d'une telle situation, qui serait que les éducateurs découvrent, grâce à leur pensée critique, un ordre resté caché à l'éducateur (maître nageur) compétent. La pensée critique ne saurait être, apparemment, qu'une pensée de survie intelligente, une pensée de bonne navigation pour une éducation guidant en sécurité le monde. Il n'en est rien. Les distinctions nécessaires (au plan conceptuel et au plan idéologique) entre pensée critique et pensée réflexive ainsi qu'entre pensée critique et activité, fonction et esprit critique sont court-circuitées comme si elles représentaient une évidence ou étaient même incluses dans les termes utilisés... Pourtant, la pensée critique et la pensée réflexive n'offrent pas un accès gratuit à la critique nécessaire des transformations.

La référence obscure à la «transformation de la réalité sociale et économique » que le citoyen critique serait capable d'entreprendre figure parmi les objectifs de la formation. Mais, formulée à l'aide d'un vocabulaire "prémâché ", elle s'apparente à une sorte de rengaine idéologique pour des textes paraphilosophiques sur l'éducation. En fait, l'éducation constitue difficilement un moyen de transformation sociale mais représente plus facilement un moyen

12. Theodoridis, A. (2009) : " Humanization through liberation from the cycle of hubris: The purpose of education ", in Boudouris, K. \& Adam, M. (Eds), Greek Philosophy and the issues of our age, Vol. II, Ionia Publication, Athens, p. 218-233.

13. Voir par exemple Macgilchrist B., Myers K., Reed J. (2004) : The Intelligent School, London, Sage Publications. 
d'imposer ou de disséminer des transformations décidées à l'avance ${ }^{14}$. Le savoir "pur», celui que la communauté éducative devrait être prête à découvrir, n'est plus un savoir dangereux : la science et la pensée critique l’ont trié au préalable. Ce savoir, l'éducateur devra le légitimer auprès de l'élève qui le " (re)découvrira ", dans un esprit de socioconstructivisme mou et d'assurances positivistes. Ici encore, les textes officiels développent une rhétorique donnant à la question la forme de la réponse, afin d'engager une réflexion déjà menée qu'ils légitiment comme la seule trouvaille possible. Tout ceci relève d'une philosophie non-critique et non-réflexive qui se réfère paradoxalement aux vertus de la réflexion. Tout comme l'éducation traditionnelle le fait avec les idées révolutionnaires et radicales, en finissant par les amollir et les aplatir.

\section{LE CERCLE DE CRAIE : QUESTIONS (PHILOSOPHIQUES) POUR LA FORMATION}

D'autres critiques de la réforme peuvent s'ajouter à celle de l'usage détourné du concept de réflexion.

Le concept d'éducation est remplacé par celui d'enseignement, ce qui réduit la pédagogie à la didactique.

L'approche trop générale et stéréotypée du contexte social, économicopolitique, et culturel se développe : vertus et compétences, objectifs et méthodes, procédures et principes émergent comme des éléments neutres, universels, obligatoires, objectifs et naturels, technologiques et scientifiques, et donc inéluctables et indubitables; ils font eux-mêmes partie d'un vocabulaire européen nouveau qui tend à remplacer le texte par des mots-clés, censés véhiculer un sens clair naturellement partagé par tous.

Un vide théorique et idéologique se cache derrière la proposition purement technique décrivant la formation des enseignants : ce texte, d'abord politique, reflète une orientation politique pour l'éducation qui puise ses arguments dans les forums internationaux et européens, sans prendre en compte la spécificité des besoins nationaux présents et futurs de l'éducation grecque. Il y manque les résultats des études menées par des intellectuels et par des scientifiques sur les tendances à long terme pour l'éducation en Grèce.

Le programme reprend les orientations de ces dernières décennies : dévalorisation de la théorie, instrumentalisation constante de la pensée, " didactisation » de la pédagogie, valorisation superficielle de la dimension pratique de l'acte éducatif, tout ceci en contradiction flagrante avec la promotion permanente de la pensée critique et créative.

14. Selon Maxine Greene (1978), le souci des formateurs d'éducateurs devrait rester normatif, critique et politique. Les écoles ne peuvent ni changer l'ordre social ni légiférer pour la démocratie. Mais quelque chose peut être fait pour donner la force aux éducateurs de réfléchir sur les situations de leur vie, pour les faire parler à leur manière des manques à combler et des possibilités d'agir au nom de ce qu'ils estiment décent, humain et juste (Landscapes of Learning, New York, Teachers College Press). 
Le programme manque d'une quelconque référence à une problématisation philosophico-pédagogique. Bien sûr le rapport entre la problématisation philosophique et les ingénieries éducatives reste compliqué voire complexe ${ }^{15}$, mais les textes officiels ne tentent pas d'affronter ces contradictions et ces défis.

On constate une prolifération de généralités dans les textes d'orientation. La philosophie de l'école nouvelle, telle une philosophie préfabriquée, déclare ses principes idéologiques et théoriques sans pour autant les clarifier ni les construire systématiquement. Ne se présentant pas comme une philosophie éducative mais comme un manifeste, elle ne justifie la nécessité, pour l'enseignant grec, de modifier son rôle que pour satisfaire un besoin urgent d'adaptation aux exigences nouvelles d'une société globale et globalisante, contraignante et aliénante. Elle ne justifie l'essence de l'éducation que comme une obligation à s'adapter, sans qu'il y ait le moindre effort d'analyser la réalité à l'égard de laquelle on doit le faire : pire, en acceptant a priori cette réalité au motif de l'urgence et sous prétexte d'intérêts pratiques.

L'absence de référence claire au rôle des universités rend problématique la continuité et la cohérence entre le contenu des études initiales et celui de la formation continue. Les universités publiques demeurent les opérateurs officiels de la formation initiale des enseignants en Grèce ; ils assurent le socle qui permet la découverte, l'évaluation, l'acquisition et l'application dans les écoles des savoirs de base ; c'est aussi dans les laboratoires de recherche des universités que se développent les réflexions et les avancées scientifiques qui devraient permettre aux politiques de concevoir et de développer des politiques de formation. Cette absence de lien est source de contradictions multiples et d'apories dans la logique du programme. Cela renvoie d'autre part à un débat de fond sur le rôle de l'université dans la recherche en sciences humaines comme dans l'élaboration de la politique éducative.

L'analyse des principes fondant la logique et le droit du projet officiel sur l'éducation en Grèce doit préoccuper fortement la philosophie de l'éducation. Cette question proprement politique relève d'une possible synthèse entre les exigences internationales affectant l'éducation (économie, société, culture) et l'histoire de la civilisation et de l'éducation grecques. Ce sont là - tout comme la question des finalités de l'éducation et la redéfinition du rôle de l'éducateur - des développements à poursuivre. Il reste que le passage d'un texte politique sur l'éducation à un texte présentant une philosophie de l'éducation n'est pas simple. C'est néanmoins la première fois que l'on tente d'expliciter les fondements et l'horizon de la formation des enseignants en Grèce. Cette question intéresse l'ensemble du monde éducatif grec. En cela, c'est une question fondamentale.

15. Theodoropopulou H. (2005) : « Homo criticus, homo philosophicus », Conférence internationale, Quelle idée de l'homme pour le pédagogue, Arradon /France, 30 juin - 2 juillet $2005 ;$ (2008) : « Les miettes de relativisme : de la philosophie dans l'éducation ", in : Drouin-Hans A.-M. (coord.), Relativisme et Education, Paris, L'Harmattan, p. 67-79. 


\section{BIBLIOGRAPHIE}

CASTORIADIS C. (1997) : "La "rationalité" du capitalisme », Revue internationale de psychosociologie, Vol. III, $\mathrm{n}^{\circ} 8$.

CHARLOT B. (Dir.) (2001) : Les jeunes et le savoir : perspectives internationales, Paris, Anthropos.

DONNAY J., BRU M. (2002) : Recherches, pratiques et savoirs en éducation, Bruxelles, De Boeck.

FABRE M. (1994) : Penser la formation, Paris, PUF.

GREENE M. (1995) : "What counts as philosophy of Education? », in: Kohli W. Critical Conversations in Philosophy of Education, New York: Routledge, p. 3-23.

JONATHAN R. (1993) : "Education, Philosophy of Education and the fragmentation of value ", Journal of Philosophy of Education, Vol. 27, $\mathrm{n}^{\circ} 2$.

KAMBOUCHNER D. (2006) : "Les nouvelles tâches d'une philosophie de l'éducation », in: Le Télémaque, $\mathrm{n}^{\circ}$ 30, p. 45-65.

RENAUT A. (2008) : Quel avenir pour nos universités?, Paris, Timée.

SIMMEL G. (1978) : The Philosophy of Money, (traduction T. Bottomore \& D. Frisby), Boston: Routledge \& Kegan Paul. 